\title{
Radiation Necrosis in Pediatric Patients with Brain Tumors Treated with Proton Radiotherapy
}

\author{
(D) S.F. Kralik, C.Y. Ho, W. Finke, J.C. Buchsbaum, C.P. Haskins, and C.-S. Shih
}

\begin{abstract}
BACKGROUND AND PURPOSE: Proton radiotherapy has been increasingly utilized to treat pediatric brain tumors, however, limited information exists regarding radiation necrosis among these patients. Our aim was to evaluate the incidence, timing, clinical significance, risk factors, and imaging patterns of radiation necrosis in pediatric patients with brain tumors treated with proton radiation therapy.
\end{abstract}

MATERIALS AND METHODS: A retrospective study was performed on 60 consecutive pediatric patients with primary brain tumors treated with proton radiation therapy. Radiation necrosis was assessed by examining serial MRIs and clinical records to determine the incidence, timing, risk factors, imaging patterns, and clinical significance associated with the development of radiation necrosis in these patients. Radiation necrosis was defined as areas of new enhancement within an anatomic region with previous exposure to proton beam therapy with subsequent decrease on follow-up imaging without changes in chemotherapy.

RESULTS: Thirty-one percent of patients developed radiation necrosis with a median time to development of 5.0 months (range, 3-11 months). Risk factors included multiple chemotherapy agents ( $>3$ cytotoxic agents) and atypical teratoid rhabdoid tumor pathology $(P=$ .03 and $P=.03$, respectively). The most common imaging patterns were small (median, $0.9 \mathrm{~cm}$ ) and multifocal (63\% of patients) areas of parenchymal enhancement remote from the surgical site. The median time to complete resolution on imaging was 5.3 months (range, 3-12 months). Among patients with imaging findings of radiation necrosis, $25 \%$ demonstrated severe symptoms with medical intervention indicated.

CONCLUSIONS: Pediatric patients with brain tumors treated with proton radiation therapy demonstrate a high incidence of radiation necrosis and a short time to development of necrosis. Multiple small areas of necrosis are frequently identified on imaging. Exposure to multiple chemotherapy agents was a significant risk factor associated with radiation necrosis in these patients.

ABBREVIATIONS: $\mathrm{CTCAE}=$ Common Terminology Criteria for Adverse Events; $\mathrm{PBT}=$ proton beam radiotherapy

$\mathbf{R}$

adiation necrosis is a well-described toxicity that has been reported over a wide range of tumor pathologies and radiation doses. ${ }^{1}$ Although histopathology can be used to establish a diagnosis of radiation necrosis, more commonly a clinicoradiologic diagnosis of radiation necrosis is used to avoid surgical morbidity and potential complications. Most of the literature regarding radiation necrosis involves adult patients, but the incidence of radiation necrosis in the pediatric brain tumor population with photon radiation therapy has also been described with an inci-

Received December 8, 2014; accepted after revision January 15, 2015.

From the Departments of Radiology and Imaging Sciences (S.F.K., C.Y.H., W.F.), Radiation Oncology (J.C.B.), and Pediatrics, Hematology/Oncology Section (C.-S.S.), Indiana University School of Medicine (C.P.H.), Indianapolis, Indiana.

Please address correspondence to Stephen F. Kralik, MD, 705 Riley Hospital Dr, Indianapolis, IN, 46202; e-mail: steve.kralik@gmail.com

三 Indicates article with supplemental on-line table.

http://dx.doi.org/10.3174/ajnr.A4333 dence of $5 \%$ and is potentially exacerbated by chemotherapy so that radiation necrosis may be attributable to the combination of radiation therapy and chemotherapy. ${ }^{2,3}$ Although radiation therapy remains an important form of treatment for pediatric brain tumors, the brains of young children may respond differently to radiation therapy compared with adults. Potential etiologies reported from a limited number of animal studies have demonstrated differences in progenitor cells, local microenvironment, inflammatory response, and effects on oligodendrocytes and microglia. ${ }^{4-7}$ Consequently, there is poor understanding of the effect of radiation therapy combined with chemotherapy on the normal brain tissue of pediatric patients. Therefore, continued investigation of radiation necrosis in pediatric patients remains important to understand these differences in susceptibility to radiation injury.

Compared with conventional (photon) radiation therapy, proton beam therapy (PBT) offers the theoretic advantages of the 
absence of an exit dose, a highly conformal dose distribution, and a reduced radiation dose to adjacent normal tissue. ${ }^{8}$ Therefore, potential benefits of proton radiation therapy in patients with pediatric brain tumors may include the reduction of negative long-term effects of radiation, such as cognitive deficits, endocrine abnormalities, vascular abnormalities, and secondary malignancies. ${ }^{9}$ PBT has been used increasingly to treat pediatric brain tumors, including craniopharyngiomas, ependymomas, germinomas, and medulloblastomas; however, limited data exist regarding radiation necrosis with proton radiation therapy in this population, with only 1 small series reporting an incidence of $47 \% .^{10-14}$ The purpose of this research was to evaluate the incidence, timing, clinical significance, risk factors, and imaging patterns of radiation necrosis in patients with pediatric brain tumor with PBT.

\section{MATERIALS AND METHODS}

Following institutional review board approval, a search of the pediatric neuro-oncology patient data base at our institution identified patients with primary brain tumors who were treated with PBT, and verification of PBT treatment and doses was obtained from the radiation oncology PBT treatment data base. All patients were either those with a newly diagnosed primary brain tumor who were subsequently treated with PBT or those who had low-grade gliomas without prior treatment with radiation therapy who demonstrated tumor progression on chemotherapy necessitating treatment with PBT. Therefore, patients were excluded if there was any history of treatment with photon radiation therapy, including before PBT, concurrent with PBT, or after PBT. Patients were also excluded if there was $>1$ course of PBT. Subsequently, a retrospective review of clinical and radiologic data was performed on 60 consecutive pediatric patients with primary brain tumors who had undergone cranial PBT from January 11, 2010, to October 25, 2012, and had clinical and MR imaging follow-up performed at our institution. Patients were scanned at approximately 3 -month intervals or sooner with suspicious findings on imaging. Patients without both 6 months of clinical follow-up and MR imaging follow-up from the completion of PBT were excluded from the statistical analysis for cerebral necrosis, including if death occurred before 6 months.

PBT treatment doses followed the standard of care in the United States at a Children's Oncology Group treatment center with a continuum of radiation doses ranging from 50 to $60 \mathrm{~Gy}$ total for most. The radiation oncologist (J.C.B.) approved 3D image guidance treatment plans before every single field in real-time each day in every patient. Typical target volumes with PBT included gross tumor volume (any visible residual tumor and/or resection cavity) to a clinical target volume (area of concern) margin of $5 \mathrm{~mm}$; and the planning tumor volume (gross tumor volume plus clinical target volume) margin was set as $2 \mathrm{~mm}$ with a 5-mm margin when accounting for additional factors including smearing. All craniospinal radiation therapy was performed with PBT, not photon radiation therapy. Twenty-one patients received craniospinal radiation therapy with doses ranging from 23.4 to 36 Gy, which was performed with 1 treatment per day with no break right into the boost. Those with medulloblastoma received craniospinal irradiation while the remainder received focal PBT.
Brain MRI consisted of imaging performed with $1.5 \mathrm{~T}$ or $3 \mathrm{~T}$ (Avanto and Verio; Siemens, Erlangen, Germany) MR imaging units with axial and sagittal T1-weighted TSE, axial T2-weighted TSE, axial FLAIR, axial DWI, coronal T1-weighted TSE postcontrast with fat saturation, and axial T1-weighted MPRAGE pulse sequences. Postcontrast imaging was performed in all patients after $0.1-\mathrm{mmol} / \mathrm{kg}$ intravenous administration of gadobenate dimeglumine (MultiHance; Bracco Diagnostics, Princeton, New Jersey).

Two fellowship-trained, board-certified neuroradiologists (C.Y.H., S.F.K.) with Certificates of Added Qualification in neuroradiology independently evaluated all preoperative, immediate postoperative, and subsequent follow-up brain MRI of included patients. All sequences on preoperative and immediate postoperative brain MRI were evaluated for imaging abnormalities, with particular attention to preoperative tumor location and size, the presence of multifocal or leptomeningeal tumor, hydrocephalus, extent of tumor resection (gross total resection or subtotal resection), and immediate postoperative complications, including cytotoxic edema or hemorrhage. A gross total resection was defined as no MR imaging evidence of residual tumor on postoperative imaging. Subtotal resection was defined as any evidence of residual tumor remaining on the postoperative MR imaging. All sequences on postradiation therapy MRI were evaluated for imaging abnormalities with particular attention to new areas of parenchymal enhancement and subsequent changes of the enhancement on serial follow-up MRI. New areas of cerebral enhancement on postcontrast sequences were evaluated for locations, diameter of the largest area of enhancement, and serial changes on follow-up MRI.

Radiation necrosis was defined after consensus agreement between the 2 neuroradiologists in conjunction with a pediatric neuro-oncologist and a pediatric radiation oncologist as the following: 1) A new area of contrast enhancement occurs in the brain parenchyma, which did not demonstrate abnormal signal or enhancement before radiation therapy. 2) The enhancement must either spontaneously decrease or resolve within 6 months of development on follow-up MR imaging without additional tumor treatment intervention and without evidence of an alternate etiology (ie, stroke, hemorrhage, or infection) in conjunction with a review of clinical records performed by a board-certified pediatric neuro-oncologist. 3) The area of enhancement is confirmed to be within an area receiving a radiation dose by a board-certified pediatric radiation oncologist. Patients with subtotal resections demonstrating enlargement of residual tumor due to new areas of nonenhancement within the residual tumor followed by a spontaneous decrease in the size of the tumor were defined as having tumoral necrosis rather than radiation necrosis. Timing from the completion of PBT to the development of radiation necrosis and timing from the appearance of radiation necrosis to complete resolution of enhancement were recorded.

Among patients with imaging findings of radiation necrosis, the clinical significance of radiation necrosis was determined by a pediatric neuro-oncologist following a review of the medical records and was graded by using the Common Terminology Criteria for Adverse Events, Version 4.0 (CTCAE) grading scale for CNS necrosis seen in Table $1 .{ }^{15}$ Clinical risk factors that were 
Table 1: Common Terminology Criteria for Adverse Events, Version 4.0: central nervous system necrosis ${ }^{a}$

\begin{tabular}{cl}
\hline Grade & \multicolumn{1}{c}{ Criteria } \\
\hline 1 & $\begin{array}{c}\text { Asymptomatic; clinical, or diagnostic observations only; } \\
\text { intervention not indicated }\end{array}$ \\
2 & Moderate symptoms; corticosteroids indicated \\
3 & Severe symptoms; medical intervention indicated \\
4 & Life-threatening consequences; urgent intervention \\
& indicated \\
5 & Death \\
\hline
\end{tabular}

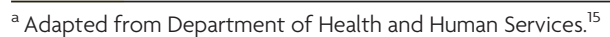

Table 2: Patient characteristics

\begin{tabular}{ll}
\hline \multicolumn{1}{c}{ Category } & \multicolumn{1}{c}{ Characteristics } \\
\hline Age & Average, 7.2 \pm 5.1 yr (range, 0.8-18 yr) \\
Sex & $14 / 52(27 \%) 3$ years of age or younger \\
Tumor pathology & Male/female, 2.5:1 \\
& Medulloblastoma and PNET ( $n=19)$ \\
& Ependymoma ( $n=12)$ \\
& Germinoma ( $n=4)$ \\
& Brain stem glioma $(n=3)$ \\
& ATRT ( $n=3)$ \\
& Craniopharyngioma $(n=3)$ \\
& Mature teratoma $(n=2)$ \\
& Pilocytic astrocytoma $(n=2)$ \\
& High-grade neuroepithelial tumor $(n=1)$ \\
& Pilomyxoid astrocytoma $(n=1)$ \\
& Pineal parenchymal tumor $(n=1)$ \\
& Chordoid meningioma $(n=1)$ \\
& Average, 54.0 Gy (range, $21-59.4$ Gy)
\end{tabular}

Note:-ATRT indicates atypical teratoid rhabdoid tumor; PNET, primitive neuroectodermal tumor.

recorded included age at the time of radiation therapy, sex, tumor pathology, tumor location, total radiation therapy dose, craniospinal radiation therapy, and chemotherapy agents. Patients who received $>3$ cytotoxic chemotherapeutic agents, not including biologic agents such as tyrosine kinase or vascular endothelial growth factor inhibitors, at any time during therapy, whether before, concurrent, or after proton beam treatment, were defined as having received "multiple chemotherapeutic agents" for the purposes of determining the statistical significance of chemotherapy agents as a clinical risk factor associated with radiation necrosis. Statistical analysis of radiation necrosis and clinical risk factors was performed by using a 2-tailed Fisher exact test. A $P$ value of $<.05$ was considered statistically significant.

\section{RESULTS}

No patients were excluded due to nondiagnostic imaging. Eight patients were excluded due to not meeting the minimum 6 months of follow-up imaging. Three of these patients (2 with diffuse infiltrative pontine gliomas and 1 with a large supratentorial primitive neuroectodermal tumor) died before 6-month follow-up imaging. Fifty-two patients were evaluated for radiation necrosis. Patient characteristics are seen in Table 2. Seventeen of $52(33 \%)$ patients had supratentorial tumors involving the pineal $(6,12 \%)$, sella $(6,12 \%)$, and parenchyma or meninges $(5,10 \%)$. Thirty-three of $52(63 \%)$ patients had infratentorial tumors with involvement of the brain stem $(3,6 \%)$ and fourth ventricle/cerebellar hemisphere (30,58\%). Multifocal tumor occurred in 2/52 (4\%). Surgical treatment included gross total resection (28,
$53.8 \%)$, subtotal resection $(23,44.2 \%)$, and none $(1,1.9 \%)$. The 1 nonsurgical case involved a brain stem tumor.

Median follow-up imaging following PBT was 18 months (average, 18.4 months; range, 6-34 months). Six patients died, including the 3 excluded patients who died before 6 -month follow up. The median time from surgery to completion of radiation therapy was 72 days (average, 104 days; range, 34-434 days). Sixteen of $52(31 \%)$ patients developed radiation necrosis as defined above with all of these patients $(16 / 16,100 \%)$ demonstrating areas of enhancement that were not directly adjacent to the resection cavity. Figures 1 and 2 show examples of patients with radiologic findings consistent with radiation necrosis. The median time to the development of radiation necrosis was 5.0 months (average, 5.5 months; range, 3-11 months). One patient demonstrated tumoral necrosis beginning at 1 month following PBT, which progressively decreased starting at 4 months following PBT. Two patients demonstrated a combination of tumoral necrosis and radiation necrosis with the tumoral necrosis in both patients beginning at 3 months following $\mathrm{PBT}$ and progressively decreasing at 9 and 12 months following PBT, respectively.

Radiation necrosis was identified with multiple tumor pathologies: medulloblastoma and primitive neuroectodermal tumor $(5 / 19,26 \%)$, ependymoma $(6 / 12,50 \%)$, atypical teratoid rhabdoid tumor $(3 / 3,100 \%)$, craniopharyngioma $(1 / 3,33 \%)$, pilocytic astrocytoma $(1 / 2,50 \%)$, and pilomyxoid astrocytoma (1/1, $100 \%$ ), while the remainder did not demonstrate radiation necrosis. Radiation necrosis was identified in multiple intracranial locations including the brain stem (56\%); cerebellum (62\%); globus pallidus and thalamus (31\%); hippocampus (13\%); and corpus callosum, periventricular white matter, and corona radiata (13\%). Multiple areas of necrosis were identified in $63 \%$ of patients, while the remainder demonstrated a solitary focus of necrosis. The median size of the largest focus of enhancement measured $0.9 \mathrm{~cm}$ (average, $1.2 \mathrm{~cm}$; range, $0.3-5.7 \mathrm{~cm}$ ). The median time to complete resolution of all enhancing areas was 5.3 months (range, 3-12 months), with complete resolution of enhancement seen in $50 \%$ of patients at 3 months, in $75 \%$ of patients at 6 months, and in $100 \%$ of patients at 12 months.

Clinical risk factors associated with radiation necrosis are listed in Table 3. Patients who received multiple chemotherapeutic agents and atypical teratoid rhabdoid tumor pathology were more likely to develop radiation necrosis $(P=.03$ and $P=.03$, respectively). Chemotherapy details are demonstrated in the Online Table. Five patients who received multiple chemotherapeutic agents before PBT developed radiation necrosis, while 3 patients who received multiple chemotherapeutic agents before PBT did not develop radiation necrosis. No patients received $>3$ chemotherapeutic agents during PBT. Among patients with imaging findings of radiation necrosis, $12 / 16(75 \%)$ were categorized as having grade 1 (asymptomatic) and 4/16 (25\%) had grade 3 (severe symptoms) according to the CTCAE central nervous system necrosis grading scale. One patient with severe symptoms was treated with hyperbaric oxygen therapy. Among all patients treated with PBT, symptomatic radiation necrosis was present in $4 / 52(7.7 \%)$. 

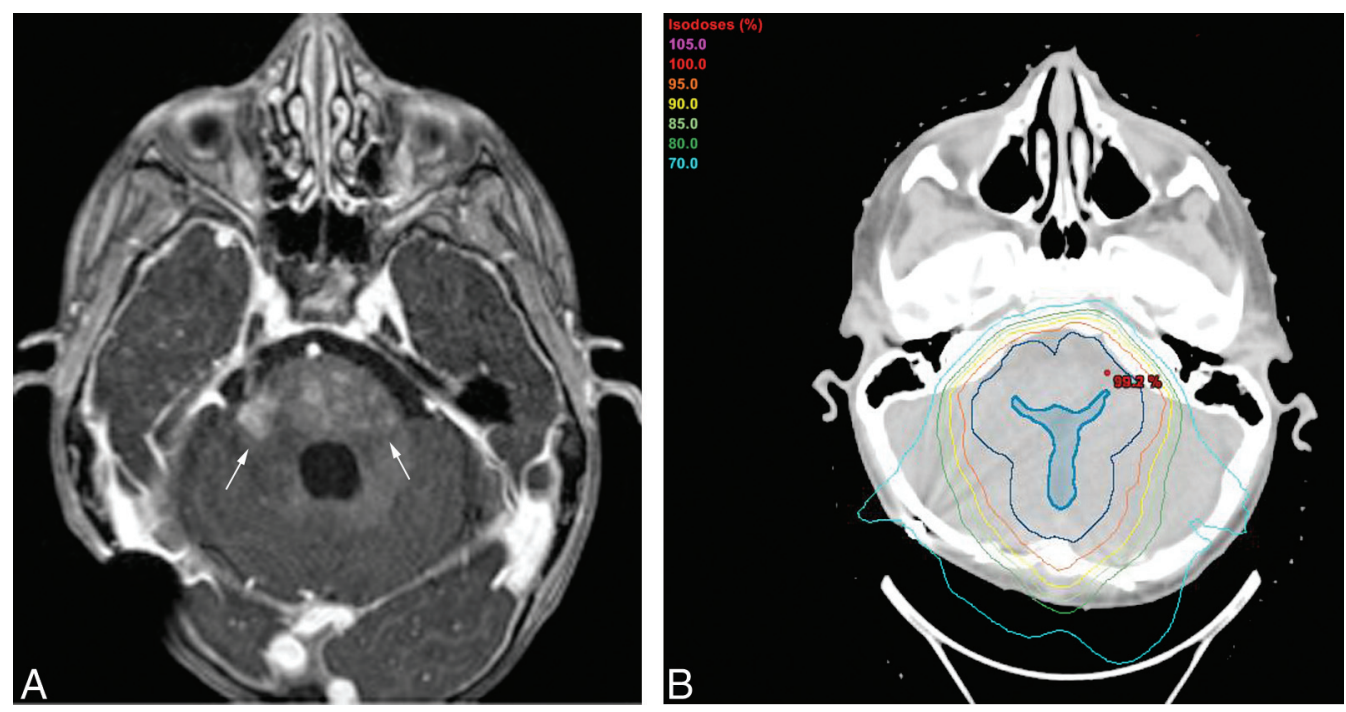

FIG 1. A 2-year-old child with a posterior fossa ependymoma status post gross total resection who developed multiple small foci of abnormal enhancement (arrows) in the pons and middle cerebellar peduncles, seen on an axial TIWI+C image $(A)$, located within the radiation field ( $B)$ at 6 months following completion of PBT. Shown in the radiation treatment image $(B)$ are the target structures of the gross tumor volume (dark blue filled) and the clinical target volume (darker blue, not filled). The dose lines of the proton beam treatment plan (analogous to the elevation lines of a topographic map) are shown as percentages of the prescription dose (59.4 Gy) in light purple (105\%), red (100\%), orange (95\%), yellow (90\%), light green (85\%), forest green (80\%), and cyan (70\%).
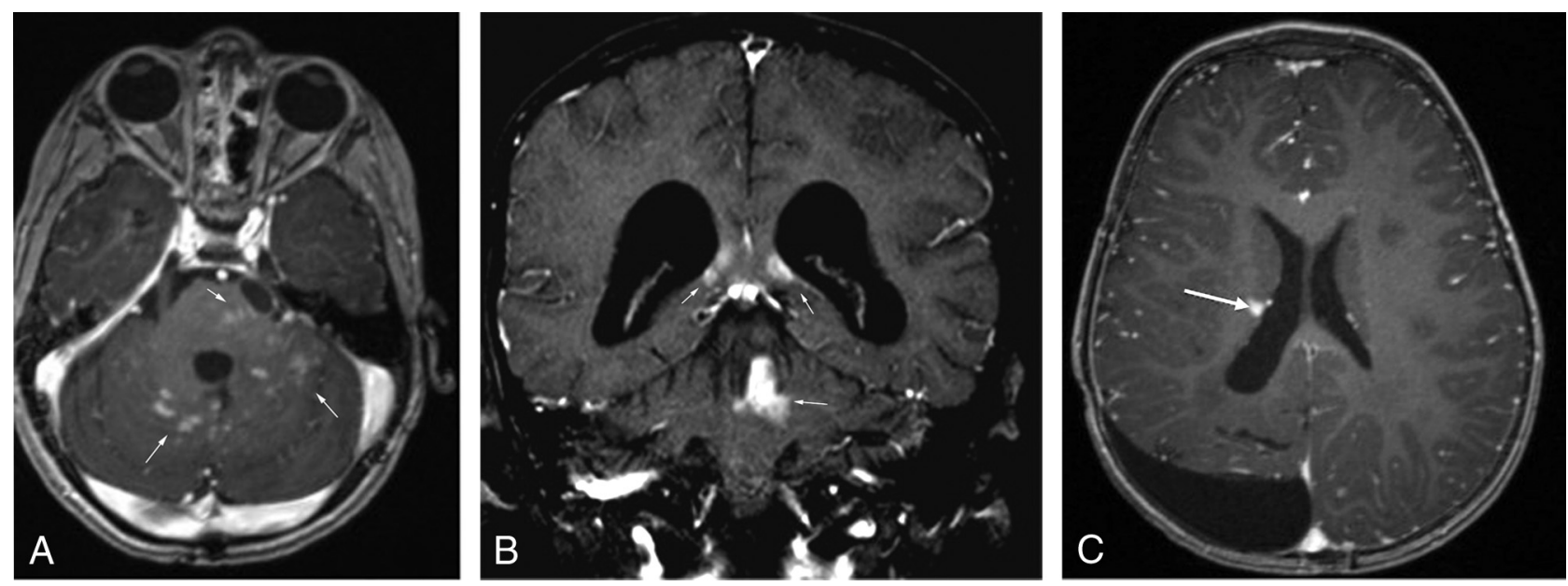

FIG 2. Examples of radiation necrosis in patients with pediatric brain tumor treated with proton radiation therapy. A, A 4-year-old child with a posterior fossa ependymoma status post subtotal resection who developed multiple small foci of abnormal parenchymal enhancement (arrows) in the pons and cerebellum seen on an axial TIWI+C image at 4 months following completion of PBT. B, A 7-year-old child with a posterior fossa medulloblastoma status post subtotal resection who developed multiple small foci of abnormal parenchymal enhancement (arrows) in the corpus callosum/periventricular white matter and the cerebellum and left superior cerebellar peduncle seen on a coronal TIWI+C image at 7 months following completion of PBT. C, A 2-year-old child with a supratentorial ependymoma status post gross total resection who developed a single small foci of abnormal parenchymal enhancement (arrow) in the right periventricular white matter seen on an axial TTWI +C image at 11 months following completion of PBT.

\section{DISCUSSION}

The prospective diagnosis of radiation necrosis in patients with brain tumor remains challenging and strongly reliant on clinical and radiologic data because biopsy of new imaging findings is uncommon, particularly in pediatric patients who may develop new lesions in critical structures such as the brain stem. Because a myriad of imaging appearances have been described with radiation necrosis, prospectively diagnosing radiation necrosis relies on a multitude of factors, including the imaging appearance and timing of necrosis and the larger clinical picture from the multidisciplinary care of these patients. In most cases, the confirmation of radiation necrosis is the result of the serial clinical and radio- logic follow-up of these patients. The imaging pattern most frequently encountered among our patients treated with PBT was that of multifocal small areas of parenchymal enhancement not immediately adjacent to the resection cavity, which resolved at a median of 5.0 months. These findings are similar to those from a smaller series of patients treated with PBT by Sabin et al. ${ }^{10}$ The imaging pattern we identified may help radiologists avoid misdiagnosis of tumor progression or at least consider the diagnosis of radiation necrosis in pediatric patients with brain tumor with PBT.

Radiation necrosis has been more extensively evaluated in adults compared with children. The Quantitative Analyses of 
Table 3: Clinical variables associated with radiation necrosis Statistically Significant Not Statistically Significant

$>3$ Chemotherapy agents $(P=.03)$ ATRT pathology $(P=.03)$

Age, 2 years or younger $(P=.11)$

Age, 3 years or younger $(P=.34)$
$\operatorname{Sex}(P=1.0)$

Gross total surgical resection $(P=.77)$

Medulloblastoma tumor pathology $(P=.35)$

Ependymoma tumor pathology $(P=.15)$

Germinoma $^{\mathrm{a}}(P=.3)$

Infratentorial tumor location $(P=1.0)$

Pineal tumor location ${ }^{\mathrm{a}}(P=.16)$

Craniospinal radiation $(P=.48)$

Total radiation dose $(P=.66)$

Note:-ATRT indicates atypical teratoid rhabdoid tumor.

${ }^{a}$ No pineal tumors or germinomas demonstrated radiation necrosis.

Normal Tissue Effects in the Clinic group reviewed 8 adult studies, including nearly 3700 patients, and estimated a 5\% and 10\% risk of symptomatic radiation necrosis in patients who received fractionated radiation with total doses of 72 and $90 \mathrm{~Gy} .{ }^{16}$ In contrast, there are few reported large series of radiation necrosis in patients with pediatric brain tumors. In 1 study of 101 children treated with photon radiation therapy, $5 \%$ of patients developed radiation necrosis based on clinicoradiologic follow-up. ${ }^{2}$ In another study of 49 patients with malignant brain tumors treated with photon radiation therapy, high-dose thiotepa, and autologous stem cell rescue, $37 \%$ of patients developed posttreatment abnormal brain imaging findings, which were defined broadly to include contrast-enhancing lesions, T2-weighted or FLAIR hyperintense lesions, hemorrhage, or subdural fluid, though the percentage of radiation necrosis was not reported. ${ }^{3}$ In our series, $31 \%$ of patients developed radiation necrosis based on imaging, indicating a trend toward a higher incidence of necrosis with PBT compared with the incidence reported for photon radiation therapy, which was similar to the high percentage in a series of 17 patients treated with PBT, of whom 47\% developed an imaging diagnosis of radiation necrosis. ${ }^{10}$ Our results differed, however, from those of 2 separate series of pediatric patients treated with PBT for ependymoma and medulloblastoma/primitive neuroectodermal tumor, in which no cases of radiation necrosis were reported; however, neither series described the methodology for detection of radiation necrosis to determine the significance of this difference. ${ }^{12,13}$

Potential reasons for the differences in the reported incidence of radiation necrosis between photon and proton radiation therapy include differences in the clinicoradiologic definition, exclusion of patients or certain tumors, and differences in medical or radiation therapy. We chose to exclude patients who did not have 6-month follow-up imaging because our median timing to development of necrosis occurred at 5 months. We chose to require radiation necrosis to demonstrate contrast enhancement rather than any new signal abnormality on imaging, which may decrease the percentage of radiation necrosis in our series. In studies that rely on a clinicoradiologic diagnosis of radiation necrosis, there is no strict timeframe in which radiation necrosis must resolve, remain stable, or begin to decrease. A time requirement is ultimately necessary, however, to establish a basis from which a clinicoradiologic diagnosis of radiation necrosis is determined. We used a relatively conservative requirement that the enhancement resolve or begin to decrease at 6 months, and this may lower the incidence of radiation necrosis in our patients. Last, studies relying on a clinicoradiologic diagnosis of radiation necrosis without histopathology cannot be directly compared with studies describing radiation necrosis on the basis of histopathology. In our study, we cannot definitively conclude that these areas of enhancement represent radiation necrosis on histopathology versus an alternate process that is exacerbated by the effects of chemotherapy.

Pseudoprogression is one such potential consideration for an alternate process occurring in these patients. The pathophysiology of pseudoprogression is poorly understood, and there is overlap in both terminology and appearance with radiation necrosis; however, tissue obtained from patients with pseudoprogression does not demonstrate the same findings seen in radiation necrosis. ${ }^{17}$ Pseudoprogression typically is defined as occurring within 3 months after completion of treatment but can range up to 6 months after therapy completion. ${ }^{17,18}$ In addition to relatively late timing encountered in our patients, the areas of necrosis were not within areas of resected tumor and were not within adjacent structures in which tumor growth would characteristically occur, both of which are atypical for pseudoprogression. Therefore, the current definition, description, and understanding of pseudoprogression do not provide an adequate explanation of our findings. If one recognizes the potential effects of chemotherapy in conjunction with PBT and the lack of histologic proof in our patients, "treatmentrelated cerebral necrosis" may be a more preferable description of the findings among our patients and more indicative of potential multifactorial causes than radiation alone. Ultimately, the major point of emphasis is that PBT may result in a significant degree of necrosis and the effects of PBT may be potentiated by additional factors, particularly chemotherapy.

The potential for chemotherapeutic interactions with radiation therapy has been well-documented, albeit not well-understood. For example, as chemosensitizers, temozolomide and bevacizumab have been used concurrently with radiation therapy in high-grade gliomas, and carboplatin has been studied as a radiation sensitizer with medulloblastomas and other CNS tumors. ${ }^{19-22}$ In addition, chemotherapeutics such as anthracycline and doxorubicin are commonly avoided during radiation and can even remotely result in radiation recall with a later insult. ${ }^{23} \mathrm{Fi}-$ nally, there are many chemotherapeutics that may have direct CNS toxicity such as methotrexate and ifosfamide, which could potentiate CNS radiation necrosis. With this in mind, the presence, timing, and dosage of single or multiple chemotherapeutic agents in conjunction with radiation may influence the incidence and severity of radiation necrosis. Although we are not able to implicate specific agents in our study, due to different treatment protocols for different tumors, we are able to show that the presence of multiple chemotherapeutic agents significantly increases the risk of radiation necrosis in our study. Fifty percent of the patients in this study received multiple chemotherapeutic agents and therefore compose a large percentage of our patients who 
received radiation. Furthermore, the risk factor of atypical teratoid rhabdoid tumor pathology may be related to intensive chemotherapy because all patients with atypical teratoid rhabdoid tumors received high-dose neoadjuvant chemotherapy with stem cell rescue before radiation therapy at our institution. These findings together underscore the importance of further study in understanding the interaction of chemotherapy and radiation therapy in the context of designing a brain tumor treatment plan.

Predicting the timing of radiation necrosis remains challenging, and a wide range from months to years has been reported. Understanding of the timing to development of necrosis, however, remains an important factor when imaging findings potentially representing radiation necrosis are encountered. In our series, radiation necrosis was seen at a median of 5.0 months (range, 3-11 months). This compares with radiation necrosis described at a median of 1.2 months (range, 0.5-8.0 months) and 8 months (range, 2-39 months) in 2 large series of patients with pediatric brain tumors treated with photon radiation therapy and a median time of 3.9 months in a smaller series of patients treated with PBT. $^{2,3}$ It remains uncertain which factors in patients with pediatric brain tumor account for the difference in time to development of radiation necrosis compared with adult patients, which is more typical at or greater than 12 months. ${ }^{1,24}$ Based on the proposed pathogenesis of radiation necrosis, there may be differences in the vascular endothelium, progenitor cells, oligodendrocytes, microglia, local microenvironment, and inflammatory response in the developing brain compared with adults accounting for the differences in timing. ${ }^{4-7}$ Last, differences in imaging-frequency practice patterns likely contribute to differences in the reported timing to the development of radiation necrosis, particularly because many patients may be asymptomatic. Therefore, the median time and range of the time to development of radiation necrosis encountered in our patients should be considered an approximation rather than an absolute time period.

The clinical significance of MR imaging changes suggestive of radiation necrosis is a common question for the health care provider and his or her patients. Among our series of patients with pediatric brain tumors treated with PBT who demonstrated radiation necrosis, 25\% demonstrated severe symptoms (based on the CTCAE grading scale) with medical intervention indicated. On the basis of these findings, any patient who develops similar MR imaging findings suggestive of radiation necrosis should raise high suspicion by the clinician for the current or later development of clinical symptoms, though most subjects may remain symptom-free.

Because radiation therapy remains the mainstay for many CNS tumor types, it is common for patients to receive fairly standardized maximum doses, depending on the location of the tumor. Thus, because the given radiation doses were not sufficiently heterogeneous, we were not able to document dose dependence in necrosis. However, the target doses typically used for PBT are frequently based on prescribed doses determined for photon radiation therapy. Whether similar doses using protons is required to maintain equivalent cure rates or whether lower doses with equivalent volumes may be as effective with less toxicity is not well understood.

\section{Limitations}

In our center, most patients with pediatric brain tumor are referred for proton radiation therapy because of the real or perceived benefits from PBT. However, there is still the possibility of selection bias regarding certain disease types such as glioblastoma multiforme or diffuse intrinsic pontine glioma that may be recommended for photon radiation therapy and, thus, not included in the sample. Our patient population is heterogeneous, and different tumor types necessitate different surgical or medical treatment. While we show that multiple chemotherapeutic agents increase the risk of developing radiation necrosis following PBT, our study was not able to distinguish whether any particular agent or combination of agents plays a more or less significant role. An associated limitation is the possibility that enhancing areas described in our study may represent a process that is different from the previously described histopathology of radiation necrosis and that this process is affected by chemotherapy. While the total given radiation dose did not demonstrate a statistically significant association with the development of radiation necrosis, further evaluation of the absorbed doses in areas that develop necrosis will be necessary, but this is beyond the scope of this study.

\section{CONCLUSIONS}

Pediatric patients with brain tumors treated with proton radiation therapy have a high incidence of radiation necrosis, demonstrating a short timeframe to development, which frequently occurs as multiple small areas of enhancement that are remote from the tumor site. Knowledge of the timing, incidence, and imaging appearance of radiation necrosis in patients with pediatric brain tumor treated with PBT can help with the radiologic differentiation from tumor recurrence. The presence of multiple chemotherapeutic agents was found to be a statistically significant risk factor associated with radiation necrosis. Additional analysis regarding the interaction of specific chemotherapeutic agents with PBT and the further investigation of PBT doses relative to photon radiation therapy are necessary to reduce these adverse treatment effects.

\section{REFERENCES}

1. Ruben JD, Dally M, Bailey M, et al. Cerebral radiation necrosis: incidence, outcomes, and risk factors with emphasis on radiation parameters and chemotherapy. Int J Radiat Oncol Biol Phys 2006;65:499-508

2. Plimpton SR, Stence N, Hemenway M, et al. Cerebral radiation necrosis in pediatric patients. J Pediatr Hematol Oncol 2015;32:78-83

3. Spreafico F, Gandola L, Marchiano A, et al. Brain magnetic resonance imaging after high-dose chemotherapy and radiotherapy for childhood brain tumors. Int J Radiat Oncol Biol Phys 2008;70:1011-19

4. Fukuda A, Fukuda H, Swanpalmer, J, et al. Age-dependent sensitivity of the developing brain to irradiation is correlated with the number and vulnerability of progenitor cells. J Neurochem 2005;92:569-84

5. Kalm M, Lannering B, Björk-Eriksson T, et al. Irradiation-induced loss of microglia in the young brain. J Neuroimmunol 2009;206:70-75

6. Schindler MK, Forbes ME, Robbins ME, et al. Aging-dependent changes in the radiation response of the adult rat brain. Int J Radiat Oncol Biol Phys 2008;70:826-34

AJNR Am J Neuroradiol 36:1572-78

Aug 2015 www.ajnr.org

1577 
7. Bustamante J, Czerniczyniec A, Cymeryng C, et al. Age-related changes from youth to adulthood in rat brain cortex: nitric oxide synthase and mitochondrial respiratory function. Neurochem Res 2008;33:1216-23

8. Levin WP, Kooy H, Loeffler JS, et al. Proton beam therapy. $\mathrm{Br} J$ Cancer 2005;93:849-54

9. Boehling NS, Grosshans DR, Bluett JB, et al. Dosimetric comparison of three-dimensional conformal proton radiotherapy, intensitymodulated proton therapy, and intensity-modulated radiotherapy for treatment of pediatric craniopharyngiomas. Int J Radiat Oncol Biol Phys 2012;82:643-52

10. Sabin ND, Merchant TE, Harreld JH, et al. Imaging changes in very young children with brain tumors treated with proton therapy and chemotherapy. AJNR Am J Neuroradiol 2013;34:446-50

11. Howell RM, Giebeler A, Koontz-Raisig W, et al. Comparison of therapeutic dosimetric data from passively scattered proton and photon craniospinal irradiations for medulloblastoma. Radiat Oncol 2012;7:116

12. MacDonald SM, Safai S, Trofimov A, et al. Proton radiotherapy for childhood ependymoma: initial clinical outcomes and dose comparisons. Int J Radiat Oncol Biol Phys 2008;71:979-86

13. Jimenez RB, Sethi R, Depauw N, et al. Proton radiation therapy for pediatric medulloblastoma and supratentorial primitive neuroectodermal tumors: outcomes for very young children treated with upfront chemotherapy. Int J Radiat Oncol Biol Phys 2013;87:120-26

14. MacDonald SM, Trofimov A, Safai S, et al. Proton radiotherapy for pediatric central nervous system germ cell tumors: early clinical outcomes. Int J Radiat Oncol Biol Phys 2011;79:121-29

15. Department of Health and Human Services. National Institutes of Health. National Cancer Institute. Common Terminology Criteria for Adverse Events, Version 4.0. http://www.acrin.org/Portals/0/ Administration/Regulatory/CTCAE_4.02_2009-09-15_QuickReference_ 5x7.pdf. Published May 28, 2009. Accessed November 5, 2014
16. Lawrence YR, Li XA, el Naqa I, et al. Radiation dose-volume effects in the brain. Int J Radiat Oncol Biol Phys 2010;76:S20-27

17. Parvez K, Parvez A, Zadeh G. The diagnosis and treatment of pseudoprogression: radiation necrosis and brain tumor recurrence. Int J Mol Sci 2014;15:11832-46

18. Hygino da Cruz LC Jr, Rodriguez I, Domingues RC, et al. Pseudoprogression and pseudoresponse: imaging challenges in the assessment of posttreatment glioma. AJNR Am J Neuroradiol 2011; 32:1978-85

19. Aguilera DG, Mazewski C, Hayes L, et al. Prolonged survival after treatment of diffuse intrinsic pontine glioma with radiation, temozolomide, and bevacizumab: report of 2 cases. J Pediatr Hematol Oncol 2013;35:e42-46

20. Anand AK, Chaudhory AR, Aggarwal HN, et al. Survival outcome and neurotoxicity in patients of high-grade gliomas treated with conformal radiation and temozolomide. $J$ Cancer Res Ther 2012;8:50-56

21. Floyd SR, Kasper EM, Uhlmann EJ, et al. Hypofractionated radiotherapy and stereotactic boost with concurrent and adjuvant temozolomide for glioblastoma in good performance status elderly patients: early results of a phase II trial. Front Oncol 2012;2:122

22. Jakacki RI, Burger PC, Zhou T, et al. Outcome of children with metastatic medulloblastoma treated with carboplatin during craniospinal radiotherapy: a Children's Oncology Group Phase I/II study. J Clin Oncol 2012;30:2648-53

23. Haas RL, de Klerk G. An illustrated case of doxorubicin-induced radiation recall dermatitis and a review of the literature. Neth $\mathrm{JMed}$ 2011;69:72-75

24. Peterson K, Clark HB, Hall WA, et al. Multifocal enhancing magnetic resonance imaging lesions following cranial irradiation. Ann Neurol 1995;38:237-44 